\title{
High-frequency stochastic resonance in SQUIDs
}

\author{
I.Kh.Kaufman* ${ }^{* \dagger}$, D G Luchinsky* ${ }^{* \dagger}$ P V E McClintock* ${ }^{* \ddagger}$ S M Soskin*§ and N D Stein*
}

\begin{abstract}
$\underline{\text { Abstract }}$
It is shown theoretically and by analogue electronic experiment that stochastic resonance (SR), in which a weak periodic signal can be optimally enhanced by the addition of noise of appropriate intensity, is to be anticipated in underdamped SQUIDs (superconducting quantum interference devices). It manifests under conditions quite unlike those needed for classical SR, which is restricted to low frequencies and confined to systems that are both overdamped and bistable. The zero-dispersion SR reported here can be expected over a vastly wider, tunable, range of high frequencies in highly underdamped SQUIDs that need not necessarily be bistable.
\end{abstract}

${ }^{*}$ School of Physics and Chemistry, Lancaster University, Lancaster, LA1 4YB, UK.

†Permanent address: Russian Research Institute for Metrological Service, Andreevskaya nab.2, 117965 Moscow, Russia.

${ }^{\ddagger}$ To whom corrrespondence should be addressed.

$\S$ Permanent address: Institute of Semiconductor Physics, Ukrainian National Academy of Sciences, Kiev, Ukraine. 
Stochastic resonance (SR), in which a weak periodic signal in a nonlinear system can be enhanced by an increase of the ambient noise intensity, appears to be widespread. It has been observed or invoked in contexts including geophysics [1], lasers [2], passive optical systems [3], electronic circuits and devices [4, 5], a Brownian particle in an optical trap [6], a bistable SQUID (superconducting quantum interference device) [7], a quantum 2-level system [8], arrays of SR elements [9], coexisting periodic attractors [10], threshold dynamics [11], subcritical bifurcations [12], transient dynamics [13], quasimonochromatic noise [14], crayfish mechanoreceptors [15] and excitable neurons [16]. There have recently been two topical conferences [17], several general scientific articles [18], and reviews [19, 20], including one [20] that attempts to place SR in historical context. The vast majority of this huge body of work refers to the classical two-state SR that arises in bistable systems or threshold dynamics - an inherently low frequency effect restricted to frequencies that are small in comparison with the system's reciprocal relaxation time. We now report a strong manifestation of SR in a completely different type of system. We have observed a marked noise-induced enhancement of the signal/noise ratio (SNR) for high-frequency signals in a monostable SQUID model.

The dynamics of the magnetic flux through the periodically driven SQUID loop under study can be described in terms of a resistively shunted model [21] whose governing equation, after appropriate changes of variable (see Fig. 1(a) caption), can be written

$$
\begin{aligned}
& \ddot{q}+\Gamma \dot{q}+\frac{d U}{d q}=f(t)+A \cos (\Omega t) \\
& U(q)=\frac{1}{2} B\left(q-q_{d c}\right)^{2}-\cos q \\
& \langle f(t)\rangle=0, \quad\left\langle f(t) f\left(t^{\prime}\right)\right\rangle=2 \Gamma T \delta\left(t-t^{\prime}\right)
\end{aligned}
$$

corresponding to classical motion in the potential $U(q)$ under influence of the additive noise $f(t)$. We will consider the case where the amplitude $A$ of the periodic force is small, where the constant $\Gamma$ is also small so that motion in the potential is underdamped, and where the relative magnitudes of $B$ and $q_{d c}$ are such that the potential has a single potential well, e.g. as shown in Fig.1(b).

Why should SR occur at all in a system like that described by equations (1)-(3)? The conventional, intuitively appealing, picture of SR as arising through nearly periodic fluctuation-assisted jumps between coexisting attractors (the minima of a bistable potential) is clearly inapplicable - because there is only one attractor. Theories of SR that 
are specific to bistable systems (e.g. [1, 22] are equally inapplicable. The phenomenon can, however, readily be understood in terms of the more general (universally applicable) perception of SR [5] in which it is treated as a linear response phenomenon for which a susceptibility can be defined, in close analogy [20] with the traditional treatments of other statistical phenomena in physics such as paramagnetism and electric polarisability [23]. If the SNR is defined in the usual way as the ratio of the intensity of the $\delta$-spike in the power spectrum at frequency $\Omega$ to the height of the smooth fluctuational background $Q^{(0)}(\omega)$ at the same frequency $\omega=\Omega$ then [5]

$$
\mathrm{SNR}=\frac{1}{4} A^{2}|\chi(\Omega)|^{2} / Q^{(0)}(\Omega)
$$

For equilibrium (white-noise-driven) systems, the complex susceptibility $\chi(\Omega)$ can be found from the fluctuation dissipation theorem and the Kramers-Kronig relations [23] and expressed as

$$
\begin{aligned}
\operatorname{Re}[\chi(\Omega)] & =\frac{2}{T} \mathrm{P} \int_{0}^{\infty} d \alpha\left(\frac{\alpha^{2}}{\alpha^{2}-\Omega^{2}}\right) Q^{(0)}(\alpha) \\
\operatorname{Im}[\chi(\Omega)] & =\frac{\pi \Omega}{T} Q^{(0)}(\Omega)
\end{aligned}
$$

where $\mathrm{P}$ denotes the Cauchy principal part and $Q^{(0)}(\Omega)$ is the spectral density in the absence of the periodic force. Perhaps surprisingly, the simplicity, generality and other advantages of treating SR in terms of linear response theory (LRT) did not win immediate acceptance. The approach [5] was subsequently vindicated by independent calculations [24], however, and has also led to the successful prediction of several completely new forms of SR, one of which was SR in monostable systems [25].

Equations (4) and (5) show that SR will occur in any system for which $|\chi(\Omega)|$ rises sufficiently fast with increasing noise intensity or, equivalently, whenever $Q^{(0)}(\omega)$ in a narrow range near $\omega=\Omega$ does so. In classical bistable $\mathrm{SR}$, the effect comes from the exponentially fast rise of a peak [26] in $Q^{(0)}(\omega)$ centred on zero-frequency, related to the onset of inter-attractor transitions (which is why classical SR can be considered an inherently low-frequency effect). In underdamped monostable (or bistable or multistable) oscillators that have one or more extrema in the variation of their oscillation eigenfrequency $\omega(E)$ with energy $E$, the SR arises because of the appearance of very sharp zero-dispersion peaks (ZDPs) [27] in $Q^{(0)}(\omega)$ at finite frequency that rise extremely rapidly with noise intensity. In the initial search [25] for evidence of SR in a monostable system (a tilted 
Duffing oscillator) the effect found was relatively weak: signal amplification was demonstrated but, because the SNR did not increase, many scientists did not regard the effect as being properly describable as SR. As we shall see, the SQUID model of equations (1)-(3) gives rise to a much more striking manifestation, with SNR increases that are comparable to those seen in classical bistable SR and which can in principle become arbitrarily large if $\Gamma$ is made sufficiently small.

The $\omega(E)$ dependence calculated for the SQUID equations (1)-(3) with $B=0.3$ and $q_{d c}=0$, shown in Fig.1(c), exhibits a local maximum and two local minima within the range plotted. Each of these extrema may be expected to produce a ZDP in $Q^{(0)}(\omega)$ that could in principle give rise to SR. To test this inference, we have built an analogue electronic model of equations (1)-(3) with $\Gamma=1.1 \times 10^{-3}$, using standard techniques [28]. When it was driven by quasi-white noise from an external noise generator, with $A=0$, the measured spectral density $Q^{(0)}(\omega)$ underwent dramatic changes of shape with increasing $T$, as shown in Fig. 2. The three ZDPs appeared sequentially as $T$ "tuned" the oscillator to different ranges of $\omega(E)$. When the weak periodic force $A \cos (\Omega t)$ was also added, with $\Omega$ chosen to lie close to the frequency of the local maximum of $\omega(E)$ and the corresponding ZDP where $|\chi|$ is expected to be strongly noise-dependent, the SNR was found to vary with increasing $T$ as shown by the data points of Fig. 3. At first the SNR falls, as one might expect on intuitive grounds; but there follows a range of $T$ within which the SNR markedly increases with increasing $T$, i.e. a strong manifestation of SR, before falling again at very high $T$.

The theory of these phenomena has been developed on the usual LRT basis: first, the power spectral densities $Q^{(0)}(\omega)$ in the absence of the periodic force were calculated for different values of $T$, by means of an algorithm similar to that described previously [29]; secondly, the calculated $Q^{(0)}(\omega)$ was inserted in equations $(4),(5)$ to yield the SNR, as required. The theory, shown by the full curves of Figs. 2 and 3, is in satisfactory agreement with the measurements. It demonstrates that the size of the increase in SNR is related to the magnitude of $\Gamma$ and that, if $\Gamma$ is made small enough, there is in principle no limit to the rise in SNR that can be achieved. Note also that the SQUID parameters in the present investigations have been chosen so as to provide a monostable potential mainly in order to emphasize the marked difference between zero-dispersion SR and conventional SR; but zero-dispersion SR will, of course, also occur in SQUIDs with multi-well potentials. A fuller discussion of theory and experiment will be presented elsewhere.

There are two kinds of conclusion to be drawn. A general observation is that the 
present results illustrate convincingly the beauty, power, simplicity and generality of the LRT approach [5] to SR. On this basis we have successfully predicted the occurrence of SR in the system described by equations (1)-(3) and, furthermore, have been able to derive a quantitative description of how the SNR varies with noise intensity. But the LRT perception also shows clearly that SR is not restricted to nonlinear systems of any particular type: from equations (4),(5), we can confidently anticipate SR in any equilibrium system whose spectral density of fluctuations $Q^{(0)}(\omega)$ contains sharp peaks that rise rapidly with noise intensity; closely analogous arguments have already been used to find SR in a nonequilibrium system [10].

A particular conclusion is that zero-dispersion SR is to be anticipated in underdamped SQUIDs. The optimal frequencies for the SR, i.e. the ZDP frequencies, are tunable over a very wide range - in principle running from near zero up to a maximum value not much less than the Josephson plasma frequency - by adjustment of the applied static magnetic field and/or the inductance of the loop. Although the SNR at the output of an SR (or any other) device cannot exceed that at the input [20,30], SR can still be used to improve the output SNR of signals that are obliged to pass through the device as may occur in biological systems $[15,16]$, or which originate within it as in SQUIDs where very low-level periodic magnetic fields with ambient noise induce currents directly in the loop [21]. The performance of a high-frequency SR device based on an underdamped SQUID would probably be at least comparable with that of the low-frequency SQUID-SR device already successfully demonstrated [7], and it would have the additional advantage of being tunable over a wide range of frequencies and SQUID parameters.

\section{Acknowledgements}

The research was supported by the Engineering and Physical Sciences Research Council (UK), the Royal Society of London, and the International Science Foundation. We acknowledge innumerable illuminating discussions of SR with Mark Dykman, Riccardo Mannella and Nigel Stocks. 


\section{References}

[1] R. Benzi, S. Sutera, and A. Vulpiani J. Phys. A14 (1981) L453; C. Nicolis Tellus 34 (1982) 1.

[2] B. McNamara, K. Wiesenfeld and R. Roy, Phys. Rev. Lett. 60 (1988) 2626; A. Fioretti, L. Guidoni, R. Mannella and E. Arimondo, J. Stat. Phys. 70 (1993) 403.

[3] M.I. Dykman, A.L. Velikovich, G.P. Golubev, D.G. Luchinsky and S.V. Tsuprikov, Soviet Phys. JETP Lett. 53 (1991) 193; J. Grohs, S. Apanasevich, P. Jung, H. Issler, D. Burak and C. Klingshirn, Phys. Rev. A49 (1994) 2199.

[4] S. Fauve and F. Heslot, Phys. Lett. A97 (1983) 5; L. Gammaitoni, F. Marchesoni, E. Menichella-Saetta and S. Santucci, Phys. Rev. Lett. 62 (1989) 349; V.S. Anishchenko, M.A. Safanova and L.O. Chua, Int. J. of Bifurcation and Chaos 2 (1992) 397; R.N. Mantegna and B. Spagnolo, Phys. Rev. E49 (1994) R1792.

[5] M.I. Dykman, R. Mannella, P.V.E. McClintock and N.G. Stocks, Phys. Rev. Lett. 65 (1990) 2606.

[6] A. Simon and A. Libchaber, Phys. Rev. Lett. 68 (1992) 3375.

[7] A.D. Hibbs, A.L. Singsaas, E.W. Jacobs, A.R. Bulsara, J.J. Bekkedahl and F. Moss, J. Appl. Phys. 77 (1995) 2582.

[8] R. Lofstedt and S.N. Coppersmith, Phys. Rev. Lett. 72 (1994) 1947.

[9] J.F. Lindner, B.K. Meadows, W.L. Ditto, M.E. Inchiosa and A.R. Bulsara, Phys. Rev. Lett. E75 (1995) 3; J.J. Collins, C.C. Chow and T.T. Imhoff, Nature 376 (1995) 236.

[10] M.I. Dykman, D.G. Luchinsky, R. Mannella, P.V.E. McClintock, N.D. Stein and N.G. Stocks, Soviet Phys. JETP Lett. 58 (1993) 150.

[11] Z. Gingl, L.B. Kiss and F. Moss, Europhys. Lett. 29 (1995) 191.

[12] S.T. Vohra and F. Bucholtz, J. Stat. Phys. 70 (1993) 413.

[13] J. Iwaniszewski, Nuovo Cimento D 17 (1995) 819.

[14] A. Neiman and L. Schimansky-Geier, Phys. Rev. Lett. 72 (1994) 2988.

[15] J.K. Douglass, L. Wilkens, E. Pantazelou and F. Moss, Nature 365 (1993) 337. 
[16] A. Longtin, Nuovo Cimento D 17 (1995) 835.

[17] F. Moss, A.R. Bulsara and M.F. Shlesinger (eds), J. Stat. Phys. 70 (1993) 1-512; A.R. Bulsara, S. Chillemi, L.B. Kiss, P.V.E. McClintock, R. Mannella, F. Marchesoni, C. Nicolis and K. Weisenfeld (eds), Nuovo Cimento D 17 (1995) 653-987.

[18] K. Wiesenfeld, Physics World, February 1993, 23; F. Moss and Xing Pei Nature 376 (1995) 211; F. Moss, Scientific American 273 (1995) 49; A.R. Bulsara and L. Gammaitoni, Physics Today, March 1996, 39.

[19] P. Jung, Phys. Rep. 234 (1993) 175; F. Moss, in Contemporary Problems in Statistical Physics (ed. Weiss, G. H.) 205-253 (SIAM, Philadelphia, 1994); K. Weisenfeld and F. Moss, Nature 373 (1995) 33.

[20] M.I. Dykman, D.G. Luchinsky, R. Mannella, P.V.E. McClintock, N.D. Stein and N.G. Stocks, Nuovo Cimento D 17 (1995) 661.

[21] R. Barone and G. Paterno, Physics and Applications of the Josephson Effect (Wiley, New York, 1982).

[22] Hu Gang, G. Nicolis and C. Nicolis, Phys. Rev. A42 (1990) 2030; P. Jung and P. Hanggi, Phys. Rev. A44 (1991) 8032.

[23] L.D. Landau and E.M. Lifshitz, Statistical Physics (Pergamon, New York, 1980).

[24] M. Morillo and J. Gomez-Ordonez, Phys. Rev. Lett. 71 (1993) 9.

[25] N.G. Stocks, N.D. Stein and P.V.E. McClintock, J. Phys. A 26 (1993) L385.

[26] M.I. Dykman, M.A. Krivoglaz and S.M. Soskin, in Noise in Nonlinear Dynamical Systems, eds. F. Moss and P.V.E. McClintock, vol.2, 347-380 (Cambridge University Press, 1989).

[27] S.M. Soskin, Physica A 155 (1989) 401; S.M. Soskin, Physica A 180 (1992) 386; P.V.E. McClintock, S.M. Soskin, N.D. Stein and N.G. Stocks, Phys. Rev. E48 (1993) 147.

[28] L. Fronzoni, in Noise in Nonlinear Dynamical Systems, eds. F. Moss and P.V.E. McClintock, vol.3, 222-242 (Cambridge University Press, 1989); P.V.E. McClintock and F. Moss, F. in loc.cit. 243-274. 
[29] M.I. Dykman, R. Mannella, P.V.E. McClintock, S.M. Soskin and N.G. Stocks, Phys. Rev. A42 (1990) 7041.

[30] M. De Weese and W. Bialek, Nuovo Cimento D 17 (1995) 733. 


\section{Figures}

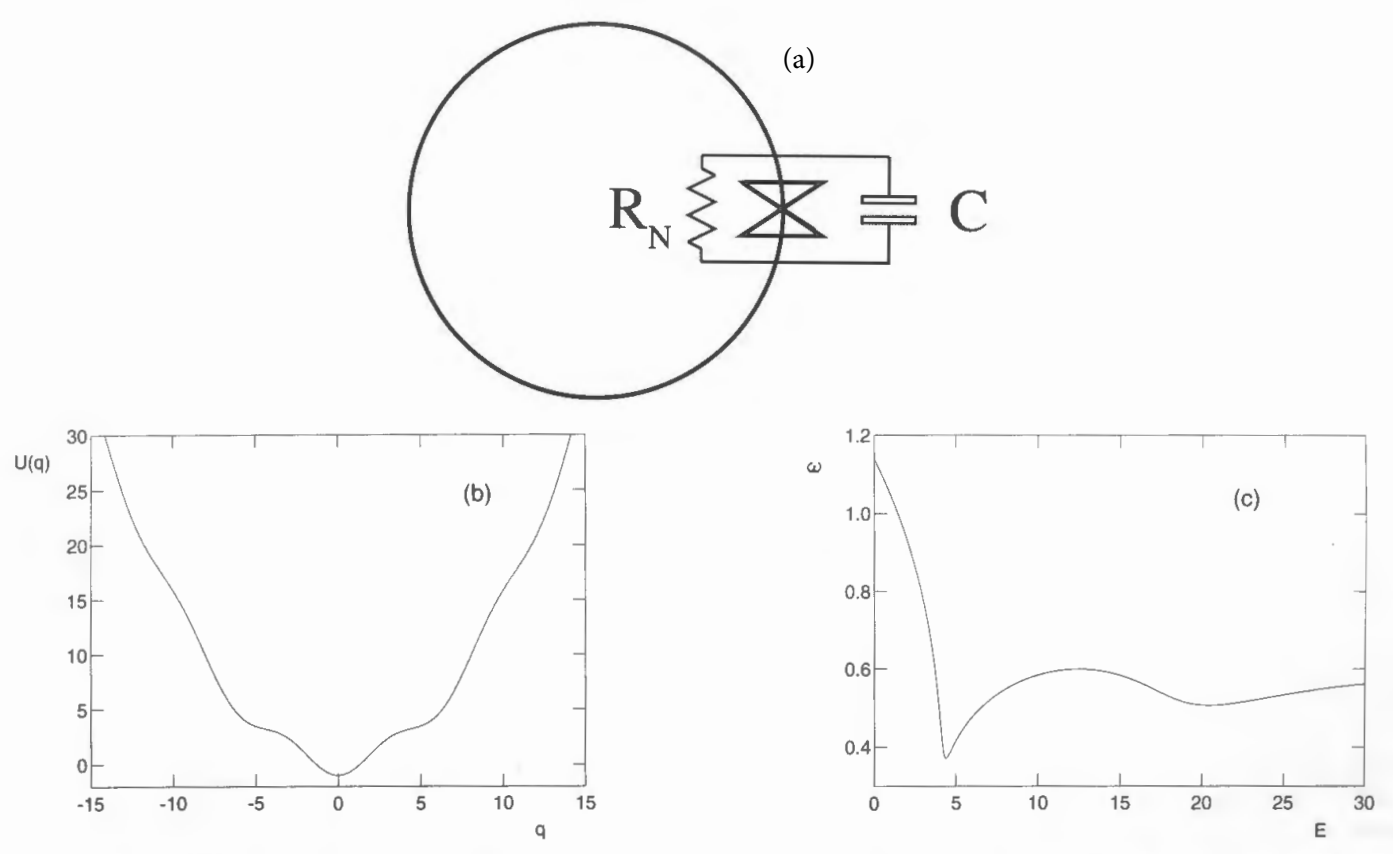

Figure 1: (a) The SQUID consists of a closed loop of superconductor containing a Josephson junction (weak link) indicated schematically by the cross. The time evolution of the magnetic flux $\Phi(t)$ threading the loop, can [21] be described by the equation $L C \frac{d^{2} q}{d \tau^{2}}+$ $\frac{L}{R_{N}} \frac{d q}{d \tau}+q+\beta \sin (q)=q_{e}$ where: $q=2 \pi \Phi / \Phi_{0} ; \quad q_{e}=2 \pi \Phi_{e} / \Phi_{0} ; \quad \beta=2 \pi L J_{c} / \Phi_{0} ; \quad \Phi$ is the full magnetic flux through the loop; $\Phi_{e}$ is the flux of the external magnetic field; $\Phi_{0}=h / 2 e$ is the flux quantum; $L$ is the inductance of the loop; and $C, R_{N}$ and $J_{c}$ are respectively the capacitance, normal resistivity and critical supercurrent of the junction. Taking account of a noisy component $\Phi_{N}(\tau)$ (arising from noise in the external flux, to which we can formally add contributions from thermal fluctuations within the loop itself and from noise in the Josephson junction), a constant component $\Phi_{\mathrm{dc}}$, and a small periodic signal $\Phi_{s} \cos \left(\omega_{s} \tau\right)$, $\Phi_{e} \equiv \Phi_{e}(\tau)=\Phi_{d c}+\Phi_{s} \cos \left(\omega_{s} \tau\right)+\Phi_{N}(\tau),\left\langle\Phi_{N}(\tau) \Phi_{N}\left(\tau^{\prime}\right)\right\rangle=2 D \delta\left(\tau-\tau^{\prime}\right)$. Introducing the normalised variables: $\quad t=\omega_{p} \tau, \quad \Gamma=1 /\left(\omega_{p} R_{N} C\right), \quad \Omega=\omega_{s} / \omega_{p}, A=\Phi_{s} /\left(L J_{c}\right)$, $q_{d c}=2 \pi \Phi_{d c} / \Phi_{0}, T=2 \pi R_{N} D /\left(\Phi_{0} J_{c} L^{2}\right), \omega_{p}=\sqrt{\left(2 \pi J_{c}\right) /\left(C \Phi_{0}\right)}, \quad$ we obtain Eqs. (1),(2) with $B \equiv 1 / \beta$. (b) The potential of Eq.(2) with $B=0.3, q_{d c}=0$. (c) Calculated variation of eigenfrequency $\omega(E)$ with energy $E$ for the potential shown in (a). 


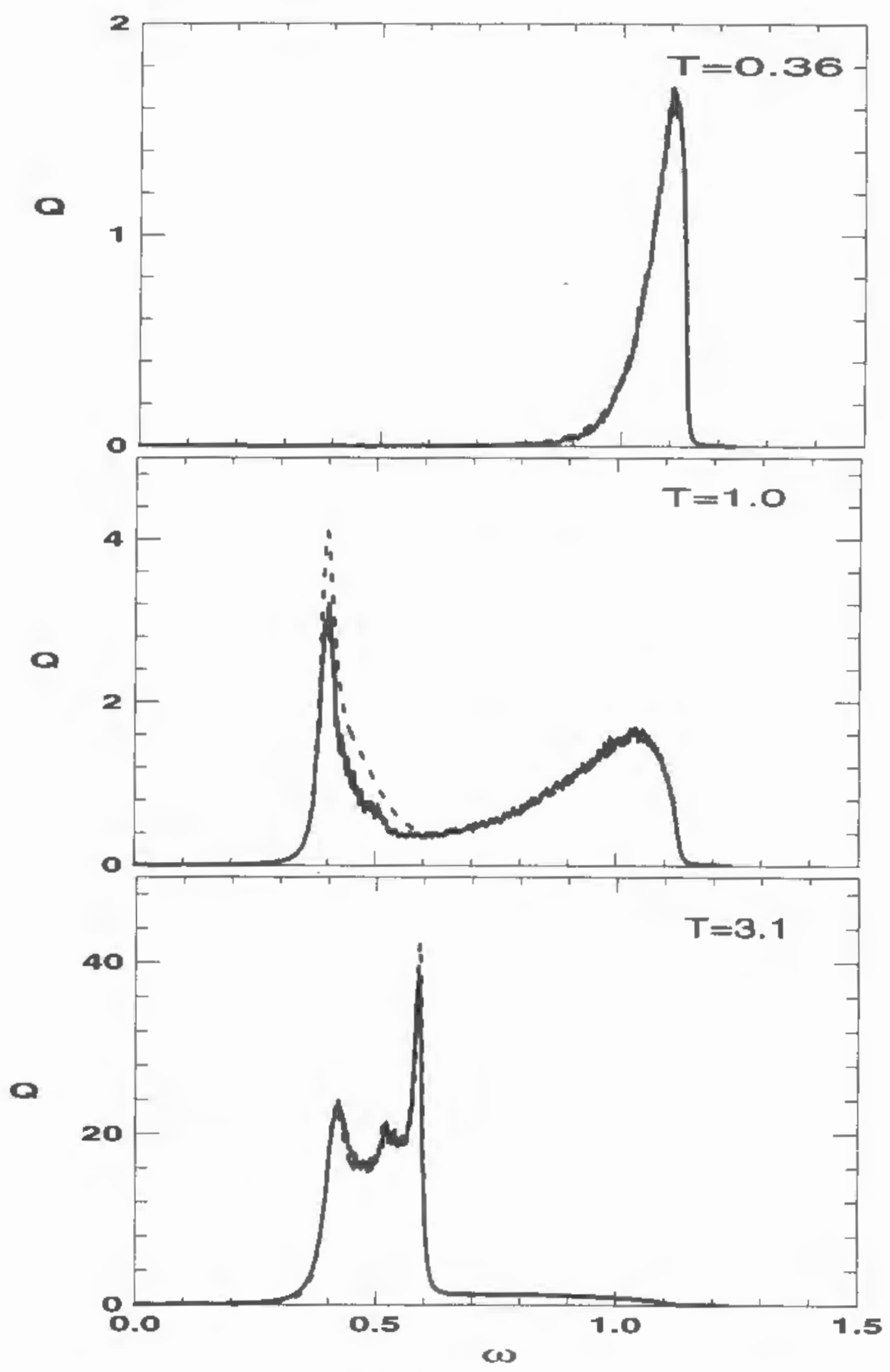

Figure 2: Spectral density of fluctuations $Q(\omega)$ measured (jagged lines) for the analogue electronic model of Eqs. (1)-(3) with $A=0, B=0.3, q_{d c}=0$, compared with the calculated behaviour (smooth curves), for three noise intensities $T$. One ZDP is seen for $T=1.0$, and three for $T=3.1$. Note the differing ordinate scales. 


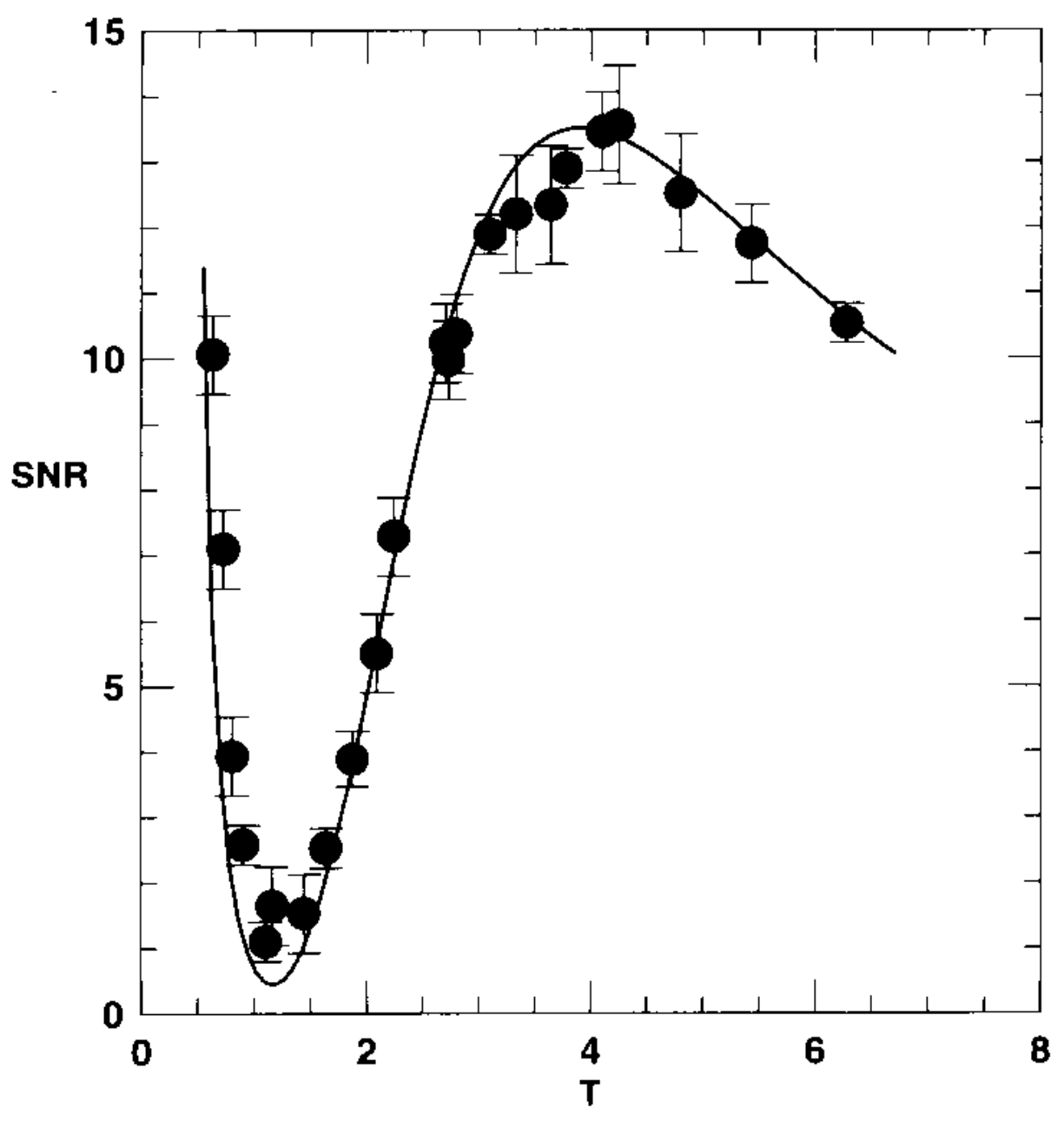

Figure 3: Signal/noise ratio (SNR) measured (data points) for the analogue electronic model of Eqs. (1)-(3) with $A=0.016, \Omega=0.62, B=0.3, q_{d c}=0$, compared with the behaviour predicted (full curve) by LRT, Eqs. (4), (5), using the calculated spectral densities $Q(\omega)$ of which three examples are plotted as dashed lines in Fig.2. 